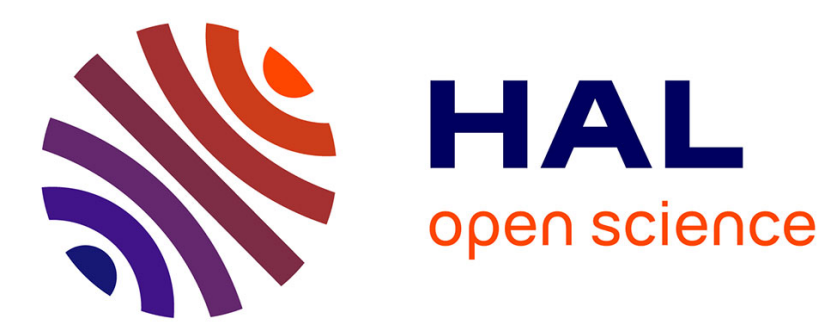

\title{
Interfacial rheology and conformations of triblock copolymers adsorbed onto the water-oil interface
}

\author{
Pablo Ramirez, Antonio Stocco, José Munoz, Reinhard Miller
}

\section{To cite this version:}

Pablo Ramirez, Antonio Stocco, José Munoz, Reinhard Miller. Interfacial rheology and conformations of triblock copolymers adsorbed onto the water-oil interface. Journal of Colloid and Interface Science, 2012, 378 (1), pp.135. 10.1016/j.jcis.2012.04.033 . hal-00708076

\section{HAL Id: hal-00708076 https://hal.science/hal-00708076}

Submitted on 14 Feb 2013

HAL is a multi-disciplinary open access archive for the deposit and dissemination of scientific research documents, whether they are published or not. The documents may come from teaching and research institutions in France or abroad, or from public or private research centers.
L'archive ouverte pluridisciplinaire $\mathbf{H A L}$, est destinée au dépôt et à la diffusion de documents scientifiques de niveau recherche, publiés ou non, émanant des établissements d'enseignement et de recherche français ou étrangers, des laboratoires publics ou privés. 


\title{
Interfacial rheology and conformations of triblock copolymers
}

\author{
adsorbed onto the water-oil interface
}

\author{
Pablo Ramírez ${ }^{\mathrm{a} *}$, Antonio Stocco ${ }^{\mathrm{b} * *}$, José Muñoz $^{\mathrm{a}}$ and Reinhard Miller ${ }^{\mathrm{c}}$ \\ ${ }^{a}$ Departamento de Ingeniería Química, Facultad de Química. Universidad de Sevilla, P. García González 1, 41012 \\ Sevilla, Spain \\ ${ }^{\mathrm{b}}$ Laboratoire Charles Coulomb, CNRS UMR 5221, Université Montpellier 2, 34095 Montpellier Cedex 5, France \\ ${ }^{c}$ Max-Planck-Institut für Kolloid- und Grenzflächenforschung, Am Mühlenberg 1, 14424 Potsdam, Germany \\ * Corresponding author. Tel.: +34 954 557180; fax: +34 954 556447. E-mail address: pramirez@ us.es (P. Ramírez) \\ **E-mail address: Antonio.Stocco@univ-montp2.fr (A. Stocco)
}

Interfacial rheology and conformations of triblock copolymers adsorbed onto the water-oil interface 


\section{Abstract}

The conformation and the dilatational properties of three non-ionic triblock PEO-PPO-PEO (where PEO is polyethyleneoxide and PPO is polypropyleneoxide) copolymers of different hydrophobicity and molecular weight were investigated at the water-hexane interface.

The interfacial behavior of the copolymers was studied by combining dilatational rheology using the oscillating drop method and ellipsometry.

From the dilatational rheology measurements the limiting elasticity values, $E_{0}$, of the Pluronics as function of surface pressure, $\Pi$, and adsorption time were obtained, i.e. $E_{0}(\mathrm{t})$ and $E_{0}(\Pi)$. Here, it is shown that $E_{0}(\mathrm{t})$ depends on the number of PEO units and on the bulk concentration, showing maximum and minimum surface elasticity values which indicate conformational changes in the interfacial layer.

Furthermore, in the framework of the polymer scaling law theory, conformational transitions were discussed in $E_{0}$ vs. $\Pi$ plots. In a dilute regime $\left(\Pi<14 \mathrm{mNm}^{-1}\right)$ at the water-hexane interface, $E_{0}=2 \Pi$ fits well all the data, which indicates a two-dimensional "stretched chain" conformation. Increasing $\Pi$, two other interfacial transitions could take place. The different behavior of Pluronic copolymers could be also described by the local minima of $E_{0}$, which depends on the hydrophobicity of the copolymers.

Conformational transitions observed by interfacial rheology were compared to ellipsometric data. Experimental results were discussed and explained on the basis of two- and threedimensional copolymer structure taking into account that PPO chains could be partially inmersed in hexane and water. 


\section{Keywords}

Pluronics, non-ionic triblock copolymer, interfacial rheology, ellipsometry, oil-water interface, conformational transitions, adsorbed layer.

\section{Introduction}

Pluronics are PEO-PPO-PEO non-ionic triblock copolymers widespread used as stabilizer for suspensions, emulsions and foams, which are needed in many industrial applications, such as cosmetics, foods, pharmaceuticals, agrochemicals, paints [1-9]. These amphiphilic copolymers (where PPO is the hydrophobic and PEO is the hydrophilic component) are also ideal model systems for the study of the interfacial transitions of complex biological macromolecules such as proteins $[10,11]$. Recently, Pluronics were also used in the fabrication of nanoparticles, capsules and other colloids in order to fabricate "smart" hybrid systems with tunable interactions at the interface and in solution $[12,13]$.

Changes in the surface structure of adsorbed polymer layers lead to significant variations in the visco-elasticity of the interfaces. The latter property and structure of Pluronics have been extensively studied at the air-water surface by means of different experimental techniques such as ellipsometry, neutron reflectivity, equilibrium and dynamic surface tension measurements and interfacial rheology [14-25]. The latter method has been shown to be a highly sensitive technique to detect conformational changes as has been reviewed in a recent paper [20].

The polymer scaling law theory $[10,26]$ has been also used to describe the interfacial behavior of Pluronics at the air-water surface $[14,15,18]$. According to this theory the variation of surface pressure with surface concentration is given by:

$\Pi \cong k_{B} T \Gamma^{y}$ 
where $k_{B}$ is the Boltzman constant, $T$ is the absolute temperature, $\Gamma$ is the surface concentration of the polymer and $y$ is a scaling parameter which describes the surface structure of the adsorbed polymer and depends on the polymer affinity to the interface, i.e. interfacial "solvent" quality. Typical values of $y$ are: $\infty$ for "poor" interfacial "solvent"; 8 for $\Theta$ interfacial "solvent"; 3 for "good" interfacial "solvent"; and 2 for stretched chains [10]. $y$ can be also obtained by interfacial rheology measurements applying the following equation:

$E_{0}=y \Pi$

where $E_{0}$ is the limiting elasticity.

Equation 2 is valid at low surface pressure values which correspond to a dilute or semi-dilute interfacial regime.

At the air-water interface, from $E_{0} v s . \Pi$ plots several interfacial transitions were observed for Pluronics. At low surface pressure (when equation 2 applies), $y=2.5-2.9$ [14,15,27], which corresponds to a flat (two dimensional) polymer conformation in a "good" interfacial "solvent". At a value of the surface pressure ca. $\Pi=5 \mathrm{mNm}^{-1}$ a maximum in the elasticity was also observed, and successively a decrease in the elasticity was associated to PEO segments protruding into the aqueous phase. Increasing $\Pi$, a new maximum of $E_{0}$ (higher than the first one) associated to a compact PEO brush structure was measured; and a final decrease of $E_{0}$ was explained as the effect of the protrusion of PPO segments into the water subphase $[14,15,22]$.

Scarce studies in the adsorption properties of these triblock copolymers have been carried out at the oil-water interface [28]. Note that a major theoretical difference between the air-water and oil-water interfaces is the fact that polymer chains out of the aqueous phase are "flattened" at the air-water, whereas at the oil-water the hydrophobic blocks can form loops in oil as the hydrophilic blocks in water [29]. Recently, Gotchev et al. has published a series of papers 
studying the stability of Pluronic emulsions by means of the thin film pressure balance technique. From those studies, the authors concluded that steric interactions between PEObrushes represent one of the major effects responsible for the stabilization of Pluronic emulsions [30-32].

The goal of this investigation is to study the viscoelastic properties and conformations of adsorbed amphiphilic triblock copolymers at an oil-water interface. Three Pluronics with different hydrophobicities (which is expressed by the hydrophilic-lipophilic balance HLB) and molecular weights (which is related to the polymer conformational entropy) were investigated to better probe and understand the interfacial behavior and conformational transitions at the oilwater interface.

\section{Materials, methods and models}

\subsection{Material}

The hydrophilic-lipophilic balance value of PEO-PPO-PEO non-ionic triblock copolymers is

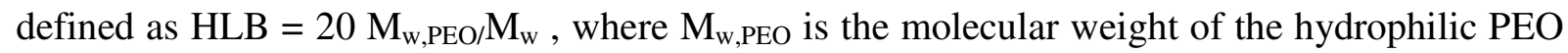
units and $\mathrm{M}_{\mathrm{w}}$ is the total Molecular weight. The triblock copolymer Pluronics F68 $\left(\mathrm{PEO}_{76^{-}}\right.$ $\mathrm{PPO}_{30}-\mathrm{PEO}_{76}, \mathrm{M}_{\mathrm{w}}=8400 \mathrm{gmol}^{-1}$ and HLB $\left.=29\right)$ and L64 $\left(\mathrm{PEO}_{13}-\mathrm{PPO}_{30}-\mathrm{PEO}_{13}, \mathrm{Mw}=2900\right.$ $\mathrm{gmol}^{-1}$ and HLB $\left.=12-18\right)$ were purchased from Sigma-Aldrich, whereas $\mathrm{P} 9400\left(\mathrm{PEO}_{21}-\mathrm{PPO}_{50^{-}}\right.$ $\mathrm{PEO}_{21}, \mathrm{Mw}=4600 \mathrm{gmol}^{-1}$ and $\mathrm{HLB}=12-18$ ) was kindly provided by BASF. The polymers were used as received.

In order to estimate the size and the overlap surface concentrations of the hydrophilic and hydrophobic blocks, it is assumed that PPO in oil and PEO in water are in "good" solvents. 
Ramírez Page 6

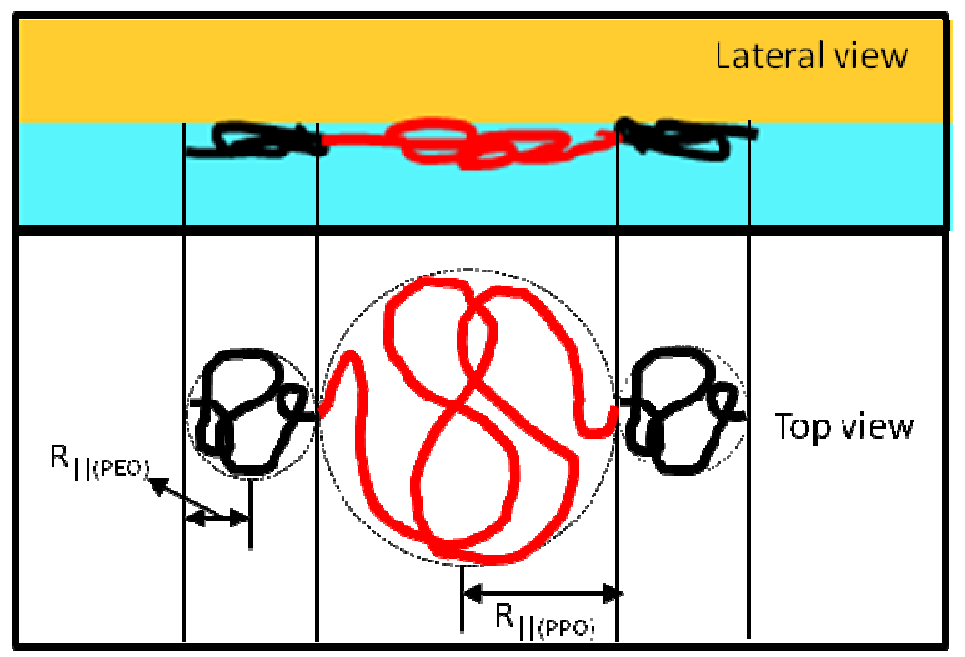

Figure 1. Lateral and top view of the model proposed to calculate the overlap surface concentration $\left(\Gamma_{2 D}\right)$, for the triblock copolymers in a two dimensional conformation.

For a flat two dimensional copolymer conformation, a first overlap surface concentration, $\Gamma_{2 D}$ $\left(\mathrm{mg} / \mathrm{m}^{2}\right)$, can be defined as:

$\Gamma_{2 D}=\frac{\left(M_{w} / N_{A v}\right)}{A_{2 D}}$

where $M_{w}$ is the molecular weight of the polymer, $N_{A v}=6.022 \cdot 10^{23} \mathrm{~mol}^{-1}$ and $A_{2 D}$ is the area occupied by the polymer in a $2 \mathrm{D}$ conformation at the interface. $A_{2 D}$ is calculated by the following equation:

$\left.\left.A_{2 D}=2 \pi R_{\mathrm{II}(P E O)}\right)^{2}+\pi R_{\mathrm{II}(P P O)}\right)^{2}$

where $R_{\|(\mathrm{PEO})}=l_{\mathrm{PEO}} N_{P E O}^{3 / 4}$ and $R_{\|(\mathrm{PPO})}=l_{\mathrm{PPO}} N_{P P O}^{3 / 4}$ (where $N_{i}$ is the number of repeating units and $l_{i}$ is the persistence length of the polymer units). $l_{\mathrm{PEO}}=0.24 \mathrm{~nm}$ and $=l_{\mathrm{PPO}}=0.51 \mathrm{~nm}$ was assumed for the three copolymers $\mathrm{PEO}_{N_{P E O}}-\mathrm{PPO}_{N_{P P O}}-\mathrm{PEO}_{N_{P E O}}[18]$. Figure 1 shows the lateral and top view of the copolymer in the flat 2D conformation. Two other overlap surface 
concentrations could be defined when the polymer blocks are no longer flat at the interface and start to protrude in the subphase:

$\Gamma_{P P O}=\frac{\left(M_{w} / N_{A v}\right)}{\pi R_{F, P P O}^{2}}$ and $\Gamma_{P E O}=\frac{\left(M_{w} / N_{A v}\right)}{2 \pi R_{F, P E O}^{2}}$

where $\mathrm{R}_{\mathrm{F}, \mathrm{PPO}}$ and $\mathrm{R}_{\mathrm{F}, \mathrm{PEO}}$ are the three dimensional Flory radii of PPO and PEO, i.e. $\mathrm{R}_{\mathrm{F}}=l N^{3 / 5}$ (for "good" solvent). The two latter surface concentrations (equations 4 and 5) correspond to the overlap of the PPO and PEO blocks in a mushroom's conformation respectively. In Table 1 the molecular weight and the overlap surface concentrations for the three copolymers are reported.

\section{Table 1}

The solutions studied were prepared with ultrapure Milli-Q water. The concentrations of the solutions were: $\mathrm{c}=0.01,0.1,0.2,1,2,10$, and $20 \mathrm{ppm}$ for interfacial rheology measurements; and $\mathrm{c}=0.01,0.1,1,10$ and $100 \mathrm{ppm}$ for ellipsometric measurements. The critical micelle concentration of Pluronics is reported between $10^{3}$ and $\left.10^{4} \mathrm{ppm}[33,34]\right)$. The most concentrated Pluronic solutions were prepared by dissolving the proper amount of polymer by ultrasonication for 5 minutes. The measurements were carried out at room temperature. Hexane was purchased from Fluka and was purified with aluminium oxide and subsequently saturated with ultrapure Milli-Q water.

\subsection{Drop profile tensiometry}

A drop profile analysis tensiometer (PAT-1, SINTERFACE Technologies, Germany) was used for interfacial tension measurements. A hexane drop of a certain volume is formed at the capillary tip of a hooked needle inside a measuring glass cell containing the aqueous polymeric solution. The drop images are recorded and from its shape the interfacial tension can be calculated. A more detailed description can be found elsewhere [35]. The surface pressure is 
defined as $\Pi=\gamma_{0}-\gamma$, where $\gamma$ is the interfacial tension of the polymer solution against hexane, and $\gamma_{0}=51 \mathrm{mN} \mathrm{m}^{-1}$ is the interfacial tension of the pure water-hexane interface.

To study the dilatational rheology of Pluronic films a hexane drop was perturbed by small harmonic oscillations of the interfacial area leading to harmonic oscillations of the surface tension. From the Fourier transform of the response relative to the perturbation the complex visco-elastic modulus is calculated [36]:

$E(i \omega)=\frac{F[\Delta \gamma]}{F[\ln (\Delta A)]}$

This complex number can be expressed in vector form, which yields:

$E(i \omega)=E^{\prime}+i E^{\prime \prime}$

where the real part, $E^{\prime}$, is called storage modulus and coincides with the dilatational interfacial elasticity and the imaginary part, $E$ ', is called the loss modulus and it is related to the dilatational interfacial viscosity (see Supplementary Information) From the experimental data the limiting dilatational interfacial elasticity $E_{0}$ is easily obtained when the dilatational interfacial viscosity is negligible. The latter condition is true for insoluble films or if the drop area oscillation is much faster than the characteristic time of the relaxation processes.

In order to explore the elasticity of the adsorbed Pluronic films with increasing the total amount of polymer at the interface, small harmonic oscillation of the area of the hexane drop with a frequency of $0.2 \mathrm{~Hz}$ was imposed along the adsorption process at concentrations ranging from 0.01 to $20 \mathrm{ppm}$. Once the equilibrium was reached harmonic oscillations of different frequencies were carried out. 
Diffusional model. According to the diffusional model [36,37] both storage and loss modulus are functions of two parameters, the limiting elasticity, $E_{0}$, and the characteristic frequency, $\omega_{0}$, as indicated by the following equations:

$E^{\prime}=E_{0} \frac{1+\zeta}{1+2 \zeta+2 \zeta^{2}}$

$E^{\prime \prime}=E_{0} \frac{\zeta}{1+2 \zeta+2 \zeta^{2}}$

where $\zeta=\sqrt{\frac{\omega_{0}}{\omega}}$ and $\omega_{0}=\left(\frac{\mathrm{dC}_{\mathrm{S}}}{\mathrm{d} \Gamma}\right)^{2} \frac{\mathrm{D}}{2}, \mathrm{C}_{\mathrm{s}}$ is the bulk concentration and $\mathrm{D}$ is the diffusion coefficient.

\subsection{Ellipsometry}

Interfacial ellipsometry measures the changes in the phase $(\Delta)$ and amplitude $(\Psi)$ of polarization of the light reflected from an interface. Single angle of incidence (angle of incidence $\varphi=46^{\circ}$ ) nulling ellipsometric measurements (Multiskop, Optrel, Germany) [38] were carried out at the hexane-water interface to obtain information on the polymer structure and surface concentration. To access the liquid-liquid interface without any distortion of the optical path, a cylindrical cell (diameter and length of $7 \mathrm{~cm}$ ) was placed in the center of the goniometer with the interfacial level also adjusted in the center [39]. The polymer concentration was varied adding successively step by step into the water phase a more concentrated polymer solution after removing the same volume of the original solution. (see Supplementary Information)

The theoretical refractive indexes used in the modeling are: $\mathrm{n}$ (hexane) $=\mathrm{n}_{0}=1.3749, \mathrm{n}_{\mathrm{PEO}}=$ $1.465, \mathrm{n}_{\mathrm{PPO}}=1.448$ and $\mathrm{n}($ water $)=\mathrm{n}_{2}=1.330$. 
For interfacial thicknesses $d$ much lower than the laser wavelength $(\lambda=533 \mathrm{~nm})$, $\mathrm{d} \ll<\lambda$, ellipsometry provides just one parameter $I_{0}=\int_{-\infty}^{\infty}\left(\frac{\left(n_{L}^{2}(z)-n_{0}^{2}\right)\left(n_{L}^{2}(z)-n_{2}^{2}\right)}{n_{L}^{2}(z)}\right) d z$ which couples the information on the refractive index of the interfacial layer $\mathrm{n}_{\mathrm{L}}(\mathrm{z})$ and its thickness $(\mathrm{z}$ is the coordinate normal to the interface) [40].

$I_{0}$ is related to the measured ellipsometric phase shift $\Delta$ by:

$\Delta-\Delta_{0}=\frac{4 \pi}{\lambda} \frac{n_{0} \sin \varphi \tan \varphi}{\left(n_{2}^{2}-n_{0}^{2}\right)\left(1-\left(n_{0} / n_{2}\right)^{2} \tan ^{2} \varphi\right)} \times I_{0}$

where $\Delta_{0}$ is the measured value for the bare (hexane-water) interface [41].

To simplify the data analysis, a step-like interfacial profile was assumed:

$I_{0}=\sum_{i} \frac{\left(n_{L, i}^{2}-n_{0}^{2}\right)\left(n_{L, i}^{2}-n_{2}^{2}\right)}{n_{L, i}^{2}} d_{i}$

For any interfacial thicknesses $\mathrm{d}<<\lambda$, note that when $\mathrm{n}_{\mathrm{L}, \mathrm{i}}>\mathrm{n}_{0}, \mathrm{I}_{0}>0$ and $\Delta-\Delta_{0}>0$; whilst when $\mathrm{n}_{2}<\mathrm{n}_{\mathrm{L}, \mathrm{i}}<\mathrm{n}_{0}, \mathrm{I}_{0}<0$ and $\Delta-\Delta_{0}<0$ ( equation 10 and $\left.11, i=1\right)$.

$\Delta_{0}$ accounts both for the intrinsic profile of the interface and the surface roughness by capillary waves [42]. The effect of capillary waves on $\Delta-\Delta_{0}$ can be estimated by:

$$
I_{C W}=-\frac{3}{2} \frac{\left(n_{0}^{2}-n_{2}^{2}\right)^{2}}{n_{0}^{2}+n_{2}^{2}} \sqrt{\frac{\pi k_{B} T}{6 \gamma}}
$$

For the bare hexane-water interface $\gamma=51 \mathrm{mN} \mathrm{m}^{-1}, I_{C W}=0.001 \mathrm{~nm}$.

In order to obtain the surface concentration of the adsorbed polymer and gain some information on conformational transitions, experimental $\Delta-\Delta_{0}$ values can be compared to simulated data obtained accounting for three polymer conformations. 
Ellipsometric Models. In these models, $\Delta-\Delta_{0}$ were obtained from $I_{0}$ (eqs. 10 and 11), which was calculated for a fixed interfacial layer thickness and accounting for the refractive index of the interfacial layer $\mathrm{n}_{\mathrm{L}, \mathrm{i}}$ as a function of the polymer surface concentration $\Gamma$ (=number of chains/Area). For polymer layers, the refractive index changes linearly with the polymer volume fraction [41]. Three polymer conformations are described as follows:

1. "Flat" (two-dimensional) conformation. In this conformation the interfacial profile can be approximated by a single layer; the polymer lays on the waterside in a two dimensional state, and the thickness $d$ (equation 11) is assumed equal to $l_{\mathrm{PPO}}=0.51 \mathrm{~nm}$ and $n_{L}=\left(\Gamma l_{P P O}^{3} N_{P P O} / d\right) n_{P P O}+\left(2 \Gamma l_{P E O}^{3} N_{P E O} / d\right) n_{P E O}+\left(1-\Gamma l_{P P O}^{3} N_{P P O} / d-2 \Gamma l_{P E O}^{3} N_{P E O} / d\right) n_{2}$, where the terms in parenthesis represent the volume fractions of the polymer and solvent in the interfacial layer.

2. "Coil" conformation. The interfacial profile can be approximated by a two layers; PPO blocks protrude into hexane and PEO into water, the thicknesses of PPO in hexane and PEO in water are assumed equal to the Flory radii $\left(\mathrm{d}_{1}=R_{F, P P O}, \mathrm{~d}_{2}=R_{F, P E O}\right)[43] . I_{0}$ can be calculated as the sum of two terms accounting for a layer in hexane and one in water. $I_{0}=I_{l}\left(\mathrm{n}_{\mathrm{L} 1}, \mathrm{~d}_{1}\right)+I_{2}\left(\mathrm{n}_{\mathrm{L} 2}\right.$, $\mathrm{d}_{2}$ ), and

$$
\begin{aligned}
& n_{L 1}=\left(\Gamma l_{P P O}^{3} N_{P P O} / R_{F, P P O}\right) n_{P P O}+\left(1-\Gamma l_{P P O}^{3} N_{P P O} / R_{F, P P O}\right) n_{0}, \\
& n_{L 2}=\left(2 \Gamma l_{P E O}^{3} N_{P E O} / R_{F, P E O}\right) n_{P E O}+\left(1-2 \Gamma l_{P E O}^{3} N_{P E O} / R_{F, P E O}\right) n_{2} .
\end{aligned}
$$

3. "Mushroom" conformation , (PPO partially in hexane and water). For this conformation we assumed that only a fraction $f$ of PPO units is in hexane. In this case, $\mathrm{d}_{1}=l_{P P O}\left(f N_{P P O}\right)^{3 / 5} ; \mathrm{d}_{2}$ $=\max \left(R_{F, P E O}, 1_{\mathrm{PPO}}\left(f N_{P P O}\right)^{1 / 3}\right)$, where $\mathrm{l}_{\mathrm{PPO}}\left(f N_{P P O}\right)^{1 / 3}$ is the radius of PPO in a "poor" solvent ( water). $I_{0}=I_{1}\left(\mathrm{n}_{\mathrm{L} 1}, \mathrm{~d}_{1}\right)+I_{2}\left(\mathrm{n}_{\mathrm{L} 2}, \mathrm{~d}_{2}\right)$, and

$n_{L 1}=\left(\Gamma l_{P P O}^{3} f N_{P P O} / d_{1}\right) n_{P P O}+\left(1-\Gamma l_{P P O}^{3} f N_{P P O} / d_{1}\right) n_{0}$ 


$$
\begin{aligned}
& n_{L 2}=\left(2 \Gamma l_{P E O}^{3} N_{P E O} / d_{2}\right) n_{P E O}+\left(\Gamma l_{P P O}^{3}(1-f) N_{P P O} / d_{2}\right) n_{P P O}+ \\
& +\left(1-2 \Gamma l_{P E O}^{3} N_{P E O} / d_{2}-\Gamma l_{P P O}^{3}(1-f) N_{P P O} / d_{2}\right) n_{2}
\end{aligned}
$$

\section{Results and Discussion}

\subsection{Interfacial rheology}

Shear and dilatational interfacial rheology experiments at the hexane-water interface were carried out in order to investigate the viscoelastic properties of Pluronic copolymers. As shown in the following, dilatational interfacial rheology experiments were a very sensitive tool for our analysis; whereas, shear interfacial rheology measurements (by a biconical disk interfacial shear rheometer (Anton Paar, Germany)) were very close to the detection limit of the apparatus and could not be used in our analysis $[44,45]$.

In Figure 2, we show the frequency dependence of the real and imaginary part of the dilatational modulus for the three Pluronics at a concentration of $\mathrm{c}=0.1 \mathrm{ppm}$. For the lowest concentrations, the storage modulus is not a function of frequency and the loss modulus is close to zero. Thus, the storage modulus gives directly the value of the limiting elasticity of the adsorbed film, $E_{0}$.

For concentrations higher than $\mathrm{c}=2 \mathrm{ppm}$ the loss modulus values are no longer negligible and the storage modulus is frequency dependent at is shown for the three Pluronics in Figure 3. This change may be related with a diffusional process due to the adsorption and rearrangements of polymer chains occurring at the same time scale of the perturbation, which results in a dependence of $E^{\prime}$ and $E^{\prime \prime}$ on frequency. 


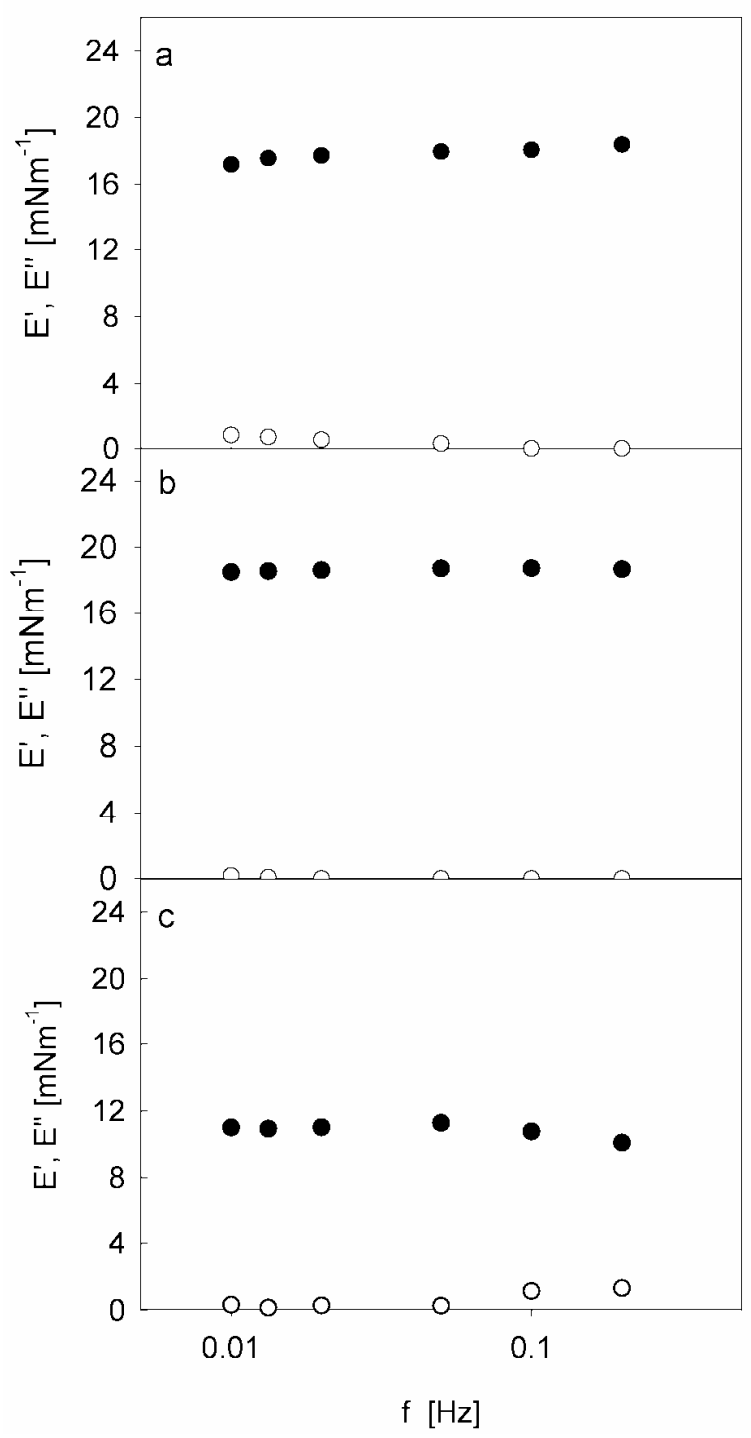

Figure 2. Storage (E', solid symbols) and loss ( $E^{\prime}$, open symbols) modulus as a function of frequency. a) Pluronic L64, c $=0.1 \mathrm{ppm}$. b) Pluronic P9400, $c=0.1 \mathrm{ppm}$. c) Pluronic LF68, $c=0.1 \mathrm{ppm}$.

Figure 3 shows the frequency dependence of $E^{\prime}$ and $E^{\prime}$ ' for the three Pluronics at the highest concentrations studied where $E_{0}$ was obtained by applying the diffusional model to the data (see section 2.2). The solid lines are the best fits to the experimental data with the $E_{0}$ and $\omega_{0}$ values given in Table 2. Equations 8 and 9 (Section 2.2) were used recently to obtain $E_{0}$ and $\omega_{0}$ for F68 and P9400 triblock copolymers at the air-water surface [33]. From the E', vs. E' plots, Hansen 
[33] showed that Pluronic F68 data fit fairly well to the model whereas the quality of the fit is somewhat lower for P9400.

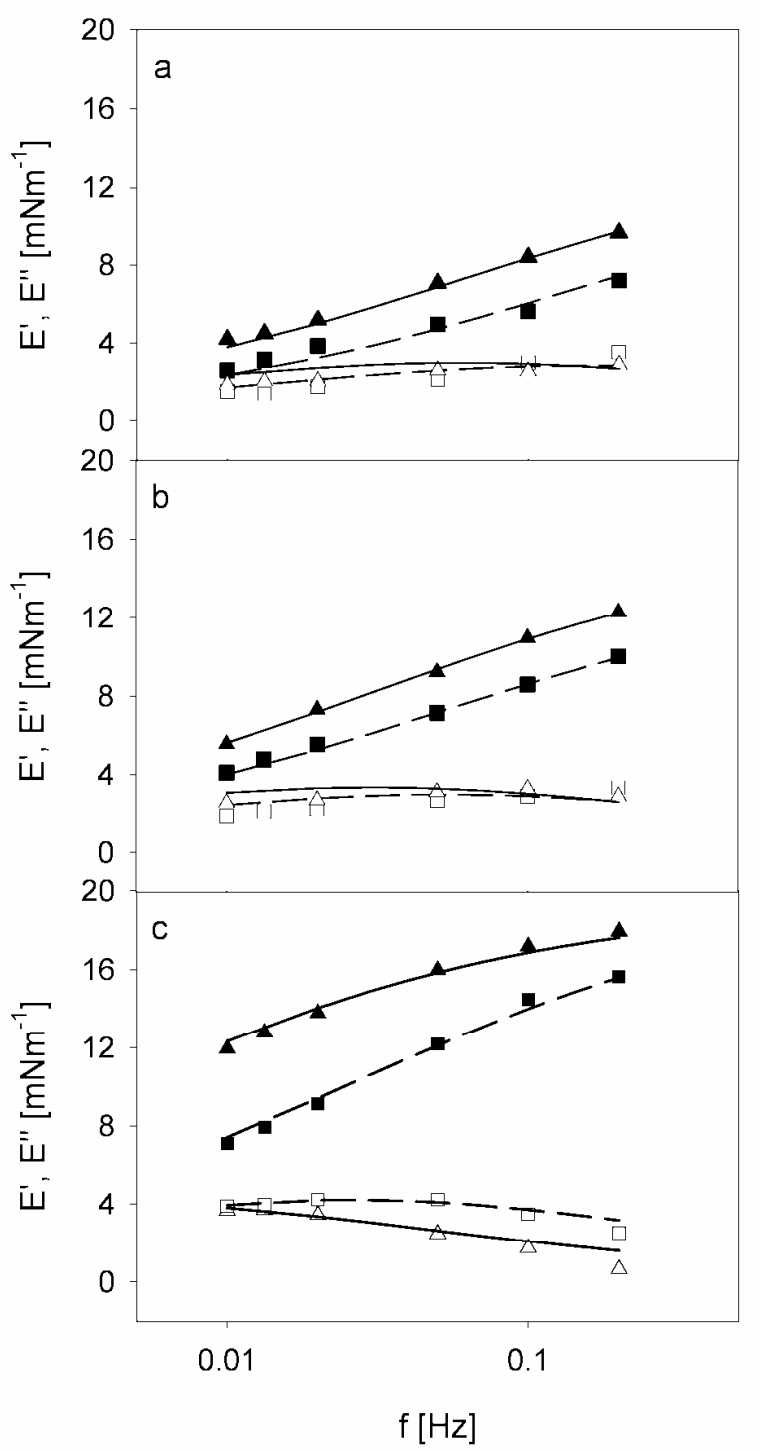

Figure 3. Storage (E', solid symbols) and loss (E', open symbols) modulus as a function of frequency. a) Pluronic L64, c = 10 ppm (triangles) and 20 ppm (squares); b) Pluronic P9400, $c$ $=10 \mathrm{ppm}$ (triangles) and $20 \mathrm{ppm}$ (squares); c) Pluronic F68, $c=10 \mathrm{ppm}$ (triangles) and $20 \mathrm{ppm}$ (squares). The lines are the best fit of the experimental data to the diffusional model with the values of $E_{0}$ and $\omega_{0}$ given in Table 1.The standard deviation of the experimental $E^{\prime}$ and $E^{\prime}$ values was about $\pm 1 \mathrm{mNm}^{-1}$ (see Supplementary Information). 
The characteristic frequency $\omega_{0}$ increases when the concentration increases, and when the molecular weight of the polymer decreases $\left(\omega_{0}(\mathrm{~L} 64)>\omega_{0}(\mathrm{P} 9400)>\omega_{0}(\mathrm{~F} 68)\right)$. Thus, $\omega_{0}$ can be regarded as the rate of the copolymer adsorption onto the interface. Note that copolymers with higher $\mathrm{M}_{\mathrm{w}}$ need more time to adsorb and rearrange onto the interface because of higher conformational entropy.

\section{Table 2}

Figure 4 shows the evolution of the surface elasticity with adsorption time for each Pluronic. The $E_{0}(\mathrm{t})$ values were obtained for Pluronics solutions ranging from $\mathrm{c}=0.01 \mathrm{ppm}$ to $\mathrm{c}=2 \mathrm{ppm}$ where the storage modulus gives directly the value of the limiting elasticity of the adsorbed film. The time evolution of the elasticity is similar for the three Pluronics. At the lowest concentration $(\mathrm{c}=0.01 \mathrm{ppm})$ an increase in the elasticity is observed. At $0.1 \mathrm{ppm}$ a maximum in the elasticity is detected with a similar value of $25 \mathrm{mNm}^{-1}$ for the three Pluronics. Furthermore, the time at which the maxima occur is also the similar, ca. 7200 s. After the latter maximum, the elasticity changes differently for the three Plutonic. For P9400 and F68, (4b and 4c, respectively) a minimum value is reached at ca. $21000 \mathrm{~s}$; and the values of these minima are: ca. $17 \mathrm{mN} \mathrm{m}^{-1}$ for P9400 and ca. $9 \mathrm{mN} \mathrm{m}^{-1}$ for F68. A minimum is not detected for Pluronic L64. Finally, at $\mathrm{c}=2$ ppm solutions (the highest concentration where the interfacial layer behaves as purely elastic), the Pluronics P9400 and L64 keep approximately the same value of the elasticity over the time of measurements, whereas for Pluronic F68 the elasticity increases until a value of ca. $17 \mathrm{mN} \mathrm{m}^{-1}$ is reached, similar to the value measured for the other two Pluronics. 


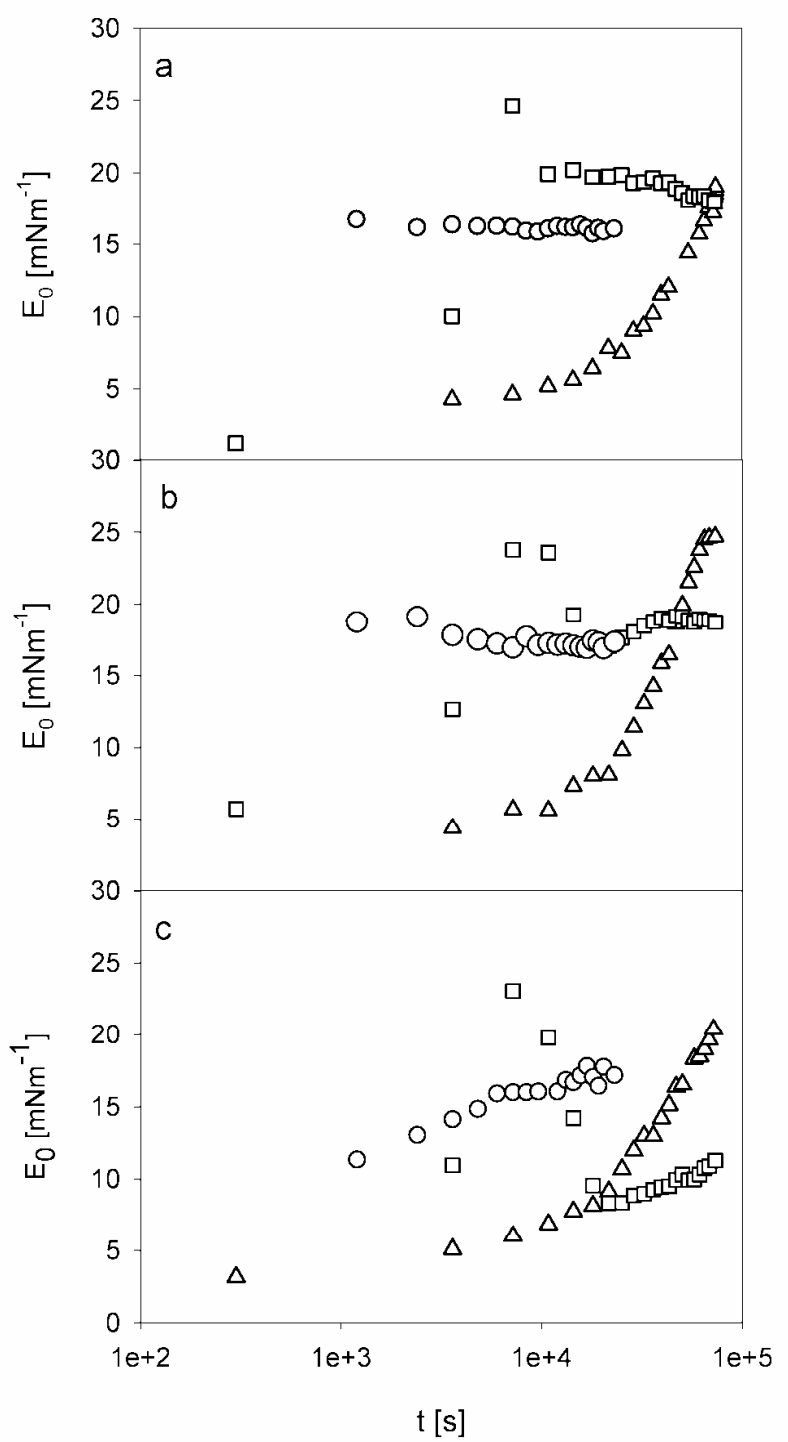

Figure 4. Limiting elasticity as a function of time. a) Pluronic L64, c =0.01 ppm (triangles), 0.1 ppm (squares) and $2 \mathrm{ppm}$ (circles); b) Pluronic P9400, $c=0.01 \mathrm{ppm}$ (triangles) $0.1 \mathrm{ppm}$ (squares) and $2 \mathrm{ppm}$ (circles); c) Pluronic F68, c $=0.01 \mathrm{ppm}$ (triangles), $0.1 \mathrm{ppm}$ (squares) and 2 ppm (circles).

As it will be discussed in the following of this section, the presence of a limiting elasticity maximum might be associated to a transition from $2 \mathrm{D}$ to $3 \mathrm{D}$ polymer conformation at the interface.

Interfacial rheology and conformations of triblock copolymers adsorbed onto the water-oil interface 
Furthermore, the decrease in the elasticity values is proportional to the number of PEO units. The copolymer with the shortest number of PEO does not show a minimum; whereas, the copolymer with the highest number of PEO shows the deepest minimum. Thus, we could relate this transition to a structural change of PEO units adopting different conformations.

In order to analyze synoptically the interfacial behavior of PEO-PPO-PEO copolymers adsorbed onto the water-hexane interface, in the framework of the polymer scaling law theory, we plotted in Figure 5 the limiting elasticity against the surface pressure for the three Pluronics. The experimental data shown in the figure involve all the bulk concentrations studied. As recently observed for Pluronics at the water-air interface [14], $E_{0}$ is only a function of the surface pressure and the same $E_{0}$ values can be obtained from different bulk concentration depending on the adsorption time.

In Figure 5, four surface pressure regions are separated by dotted lines. The first region is located between $\Pi=0$ and $14 \mathrm{~m} \mathrm{Nm}^{-1}$. In this region, the surface elasticity increases linearly with surface pressure and the line $E_{0}=2 \Pi$, fits well all the data. According to equation 2 the value of the slope $y=2$ corresponds to a "stretched chain" conformation.

It is interesting to compare the behavior of the triblock copolymers at the hexane-water interface with previous results obtained at the air-water surface $[14,15,22]$. At the hexane-water interface, the $y$ value is lower than the one obtained at the air-water surface pointing to more favorable interactions between the copolymer and the interface. Assuming that PEO interacts just with water molecules, the different behavior could be ascribed to the interactions of PPO at the interface. In fact, at the hexane-water interface PPO interactions could be more favorable than at the water-air interface, being hexane a "good" interfacial "solvent" for PPO. Hence, the first region in Figure 5 could be formed by a copolymer conformation in which both PEO and PPO 
blocks are in a two dimensional "stretched chain" conformation, resulting from the favorable interactions between the copolymer and the interface.

The elasticity values for the hexane-water interface are also higher than for the air-water interface. This difference could be also ascribed to the fact that PPO is better anchored at the water-hexane than at the water-air interface. It is also worth noting that in this region the elastic behavior of the interface is practically identical for the three Pluronics, pointing to an identical two dimensional polymer structure.

The decrease in the elasticity could occur around a polymer overlap surface concentration $\Gamma_{2 \mathrm{D}}$ (see Table 1). $y$ and $\Gamma_{2 \mathrm{D}}$ values are in fact very close for the three copolymers. According to equation 1 , the surface pressure is a function of $y$ and $\Gamma$; therefore, it is expected that the fall in elasticity starts at a similar surface pressure value for the three triblock copolymers as it is shown in Figure 5.

The second region corresponds to surface pressure values ranging from 14 to $23 \mathrm{mN} \mathrm{m}^{-1}$. For the three Pluronics a decrease in the limiting elasticity is observed. The lowering of the limiting elasticity in this region differs for the Pluronics. Pluronic L64 shows a small decrease; while on the contrary Pluronic F68 reveals a more pronounced drop. The decrease of the limiting elasticity of the Pluronic P9400 is in between the behavior of the other two Pluronics. 


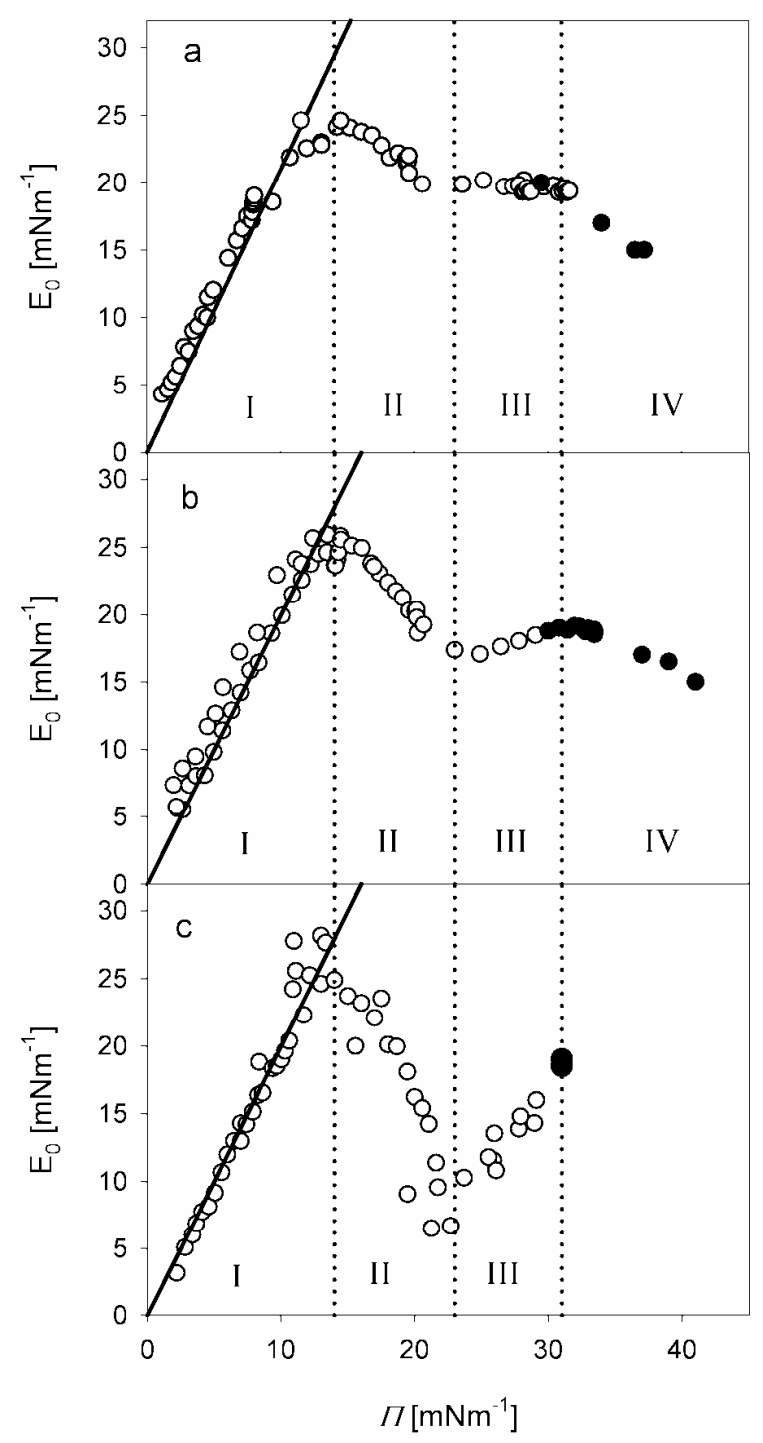

Figure 5. Limiting elasticity as a function of surface pressure. a) Pluronic L64, b) Pluronic P9400 and c) Pluronic F68. The open symbols are the data for concentrations below $c=2$ ppm where the limiting elasticity was directly obtained from the storage modulus, whereas the filled symbols account for the highest concentrations where the diffusional model has been used to determine $E_{0}$. The solid lines show a linear dependence of $E_{0}$ on $\Pi$ at low surface pressures, $E_{0}=2 \Pi$. The dotted lines separate the four different conformational regions.

As it was noticed previously in Fig. 4, the decrease of the elasticity of the adsorbed films can be explained by the occurrence of a loser structure at the interface due to a mixture of different orientations of the adsorbed Pluronics. The way how the elasticity decreases suggests a relation 
between this transition and the number of PEO units. The protrusion of PEO chains into the aqueous phase lead also to a reduction of the polymer contacts with the interface which could be explain the decrease of elasticity [14].

Between $\Pi=23$ and $31 \mathrm{mNm}^{-1}$ the elasticity increases again. This increase could be related to the formation of a more concentrated three dimensional structure ("mushroom" conformation). The elasticity rises until a value of $E_{0}=20 \mathrm{mNm}^{-1}$ for the three Pluronics. It is worth noting that in contrast with the behavior at air-water surface [14,15,22], this second maximum has a lower surface elasticity value than the first one. Therefore, at the hexane-water interface the two dimensional stretched state is more elastic than the three dimensional state. This could be due to the decrease in the polymer contacts at the interface in this region compare with the two dimension one. It was shown that at high surface concentrations multiblock copolymers adopt different conformations ("hairpins" and "loops") at the interface between two immiscible liquids [26]. The situation should be similar in the third region leading to a "mushroom conformation" of the Pluronics.

For $\Pi>31 \mathrm{mNm}^{-1}$, only the more hydrophobic polymers, L64 and P9400, (HLB = 12-18) still continue adsorbing at the interface. However, the elasticity decreases indicating that the interfacial structure is less compact. This reduction of the elasticity after the second maximum has also been observed also at the air-water interface. For the latter interface, PPO units might protrude into the aqueous phase disordering the PEO interfacial structure.

At the hexane-water interface another possible scenario is that PPO protrudes into the hexane phase reducing its contacts with the interface.

\subsection{Ellipsometry}


In order to shed some light on the four different regimes discussed in the interfacial rheology section and to obtain the polymer surface concentration, we performed ellipsometric experiments (Section 2.3, Supplementary Information) and compared the experimental data to simulations accounting for three possible polymer conformations at the interface.

For thin polymer layers at the oil-water interface, in our experimental condition $\left(\varphi=46^{\circ}\right.$, Brewster angle $=44.05^{\circ}$, section 2.3) the phase polarization change, $\Delta$, varies significantly when the surface concentration increases; in contrast, the change of the polarization amplitude $\Psi$ remains approximately constant.

Positive and negative $\Delta-\Delta_{0}$ values $\left(-0.6^{\circ}<\Delta-\Delta_{0}<0.4^{\circ}\right)$ were measured for the three copolymers (Figure 6) at different values of $\Pi$. For L64, $\Delta-\Delta_{0}=0.1^{\circ}$ at $\Pi=13 \mathrm{mN} \mathrm{m}^{-1}$. With increasing $\Pi, \Delta-\Delta_{0}$ becomes negative and after passing through a minimum it increases for $\Pi>$ $31 \mathrm{mN} \mathrm{m}^{-1}$. For P9400, a shallow minimum is also observed and for $\Pi>31 \mathrm{mN} \mathrm{m}^{-1}$ a clear increase of $\Delta-\Delta_{0}$ from ca $-0.3^{\circ}$ to ca $0.4^{\circ}$ was measured. For F68, a clear change from ca $0.3^{\circ}$ to ca $-0.6^{\circ}$ is observed occurs when $\Pi$ changes from ca 20 to $31 \mathrm{mN} \mathrm{m}^{-1}$.

For thin interfacial layers, ellipsometry provides just one parameter, $I_{0}$ (equations 10 and 11), and the information on $\mathrm{n}_{\mathrm{L}}$ and $d$ cannot be decoupled. 

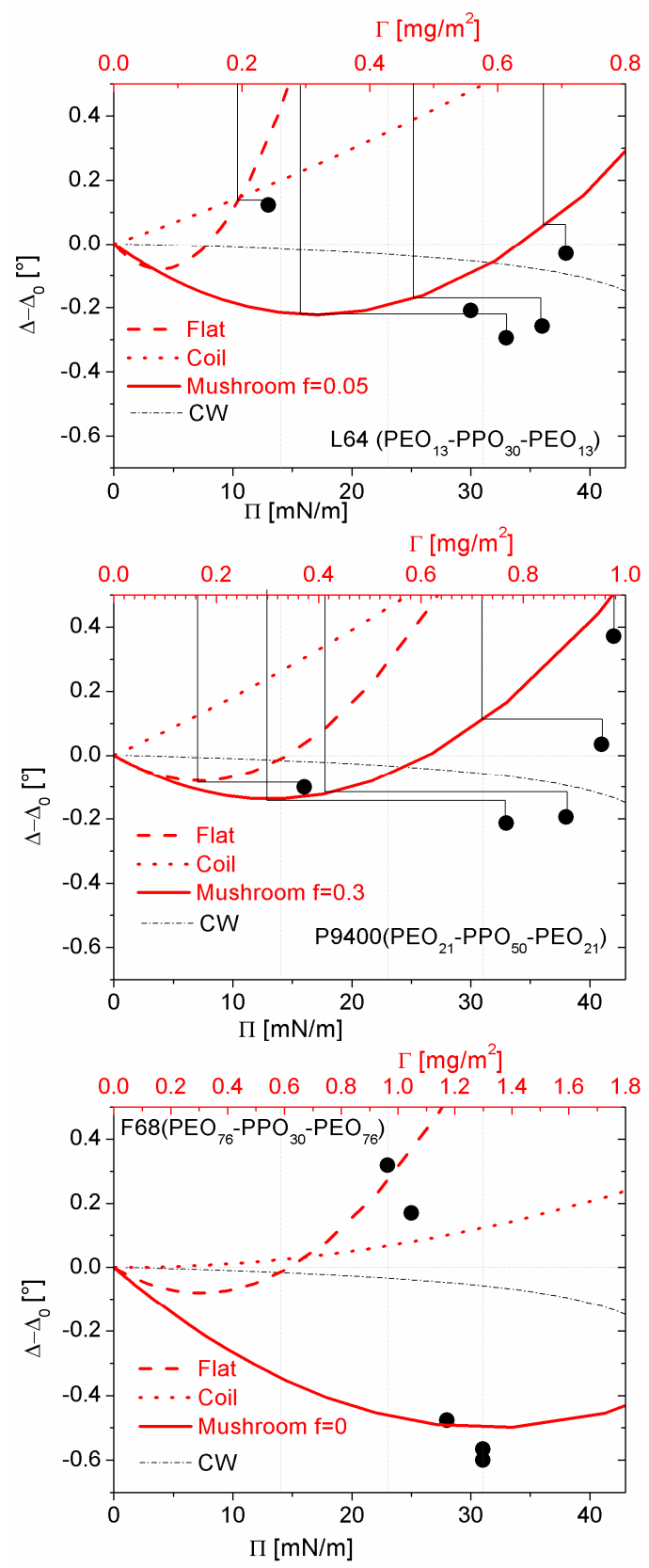

Figure 6. Experimental ellipsometric phase shift values $\Delta-\Delta_{0}$ as a function of surface pressure $\Pi$ (bottom x-axis) for L64, P9400 and F68 Pluronics. Dashed, dotted and solid (red) lines are the simulated $\Delta-\Delta_{0}$ as a function of surface concentration $\Gamma$ (top $x$-axis) for three polymer conformations: "flat", "coil" and "mushroom" accounting for a fraction $f$ of PPO in hexane respectively. The dash-dotted (black) lines are the simulated $\Delta-\Delta_{0}$ as a function of surface pressure (bottom $x$-axis) accounting for the capillary wave profile. The solid thin (black) lines help the reader to find the surface concentration of a given model starting from the experimental

Interfacial rheology and conformations of triblock copolymers adsorbed onto the water-oil interface 
data and accounting for the capillary wave contribution. The standard deviation of the experimental data was about $\pm 0.1^{\circ}$.

In the framework of a single isotropic interfacial layer profile, the non monotonous behavior of $\Delta-\Delta_{0}$ (Figure 6) could be described by an interfacial layer, in which $\mathrm{n}_{\mathrm{L}}$ varies at constant thickness. Negative $\Delta-\Delta_{0}$ values correspond to low polymer surface coverages, $\mathrm{n}_{2}<\mathrm{n}_{\mathrm{L}}<\mathrm{n}_{0}$; whereas positive $\Delta-\Delta_{0}$ values to high surface coverages, $\mathrm{n}_{\mathrm{L}}>\mathrm{n}_{0}$ (section 2.3 ). However, this profile does not account neither for the two distinguished polymer layers that might form in hexane and in water nor for the polymer composition. Note also that a single interfacial layer profile in which $d$ varies at constant $\mathrm{n}_{\mathrm{L}}$ would lead to monotonous changes of $\Delta-\Delta_{0}$ which does not describe our experimental data.

Knowing the chemical composition of the copolymers and the coil sizes in different interfacial solvent quality (Section 2.1), we evaluate the polymer surface concentration by comparing the experimental data to simulated data accounting for three different conformations and the capillary wave contribution (Section 2.3).

The capillary wave contribution to the interfacial profile was calculated using equation 12 . The latter contribution is always negative and varies from 0 to $-0.15^{\circ}$ for $\Pi=43 \mathrm{mN} \mathrm{m}^{-1}$.

We start comparing our experimental data with the simulated data for a "coil" conformation assuming that PPO is immersed in hexane and PEO in water (section 2.3). In this case, $\Delta-\Delta_{0}$ is appreciably non negative and increases monotonously. This trend can be easily understood considering that the contribution of PPO layer to $I_{0}$ is always positive since $\mathrm{n}_{\mathrm{PPO}}>\mathrm{n}_{0}$ (equation 11). PEO contribution is also positive at relative high surface concentrations; whereas its contribution can be negative at low surface concentration when the interfacial layer has an 
average refractive index $\mathrm{n}_{2}<\mathrm{n}_{\mathrm{L}, 2}<\mathrm{n}_{0}$ (equation 11). The interplay of these two contributions results in appreciable non-negative $\Delta-\Delta_{0}$ values, as shown in Fig. 6 for the "coil" conformation. As the above discussion attests, both the capillary wave profile and the "coil" conformation (or a combination of the two) cannot describe the experimental data obtained in Fig. 6. For this reason, a "flat" two dimensional polymer conformation and a "mushroom" conformation, accounting for a fraction of PPO chains immersed in hexane and in water, should be considered (Section 2.3). Assuming that the polymer conformation is two dimensional ("Flat" conformation model, section 2.3), $\Delta-\Delta_{0}$ shows a non-monotonous trend. $\Delta-\Delta_{0}$ is negative and decreases at low surface concentrations $\left(\Gamma<0.1 \mathrm{mg} \mathrm{m}^{-2}\right)$; whilst increasing $\Gamma, \Delta-\Delta_{0}$ increases becoming positive at relatively high surface concentrations. This trend reassembles the trend observed for L64 and P9400. However, the minimum $\Delta-\Delta_{0}$ in the simulation is around $-0.1^{\circ}$ whilst for L64 is $-0.3^{\circ}$ and $-0.2^{\circ}$ for P9400. For F68 the data follows a different trend with a minimum of $\Delta-\Delta_{0}$ around $-0.6^{\circ}$.

We find negative $\Delta-\Delta_{0}$ that can reproduce the experimental data only when a fraction of PPO is supposed to remain in water ("mushroom" model, PPO partially immersed in hexane and water model, section 2.3). In Fig. 6, the fraction of the $\mathrm{N}_{\mathrm{PPO}}$ repeating units in hexane, $f$, is the only adjusted parameter in a model that accounts for a polymer mushroom conformation. For the more hydrophobic system P9400, we found a good agreement between the model and the experiments for $f=0.3$, meaning that $30 \%$ of the PPO chains lay in hexane; for L64, $f=0.05$ and for the more hydrophilic system F68, $f=0$ meaning that $100 \%$ of PPO chains remain in water. Note that the increase of $f$ is consistent with an increase of polymer hydrophobicity.

This result could seem very surprising since one expects that PPO would move completely to hexane once the adsorption on the interface is completed. On the other hand, the transfer of PPO 
towards hexane is not an energy cost free process. In fact, for the transfer of PPO from water to hexane, energy should be provided to change i) the polymer hydration state, ii) the conformation and iii) to overcome the interfacial region. Note that a depleted layer is also expected at the hexane-water interface [46], which could represent an additional obstacle for the transfer of PPO into hexane.

Going back to the four regimes observed in the interfacial rheology investigation (see Fig. 5), we could now discuss some of those conformational transitions by using the ellipsometric modeling. For F68, when $\Pi>23 \mathrm{mN} / \mathrm{m}$ a transition from a "flat" two dimensional regime to a "mushroom" regime with both PEO and PPO blocks protruding in the water phase could be observed. The surface concentration around which this transition occurs is $\Gamma=\mathrm{ca} 1 \mathrm{mg} \mathrm{m}^{-2}$. Note that the transition to the latest regime, when $\Pi>31 \mathrm{mN} / \mathrm{m}$, was not observed for F68, which is the most hydrophilic system $(f=0)$.

A clear transition was instead observed for the two other copolymers L64 and P9400, which are more hydrophobic. In Fig. 6 to evaluate the surface concentrations $\Gamma$ starting from the experimental data, we draw first the vertical lines which accounts for the capillary wave contribution and the horizontal lines to find the corresponding $\Delta-\Delta_{0}$ of the ellispometric models (section 2.3).

The transition at $\Pi=31 \mathrm{mN} / \mathrm{m}$ occurs at $\Gamma=\mathrm{ca} 0.3 \mathrm{mg} \mathrm{m}^{-2}$ for both L64 and P9400 ("Mushroom" conformation, PPO partially in hexane and water" model), which is very similar to the some overlap concentrations of Table 1.

For $\Pi>31 \mathrm{mN} / \mathrm{m}, \Gamma=0.47$ and $0.67 \mathrm{mg} \mathrm{m}^{-2}$ were found for L64; and $\Gamma=0.41,0.72$ and 0.97 $\mathrm{mg} \mathrm{m}^{-2}$ for P9400. Those $\Gamma$ resulted from a model which assumes a layer thickness equal to a three dimensional Flory radius ("Mushroom" conformation, PPO partially immersed in hexane 
and water, section 2.3). However, those concentrations are already higher than the critical overlap concentrations $\Gamma_{\mathrm{PEO}}$ and $\Gamma_{\mathrm{PPO}}$ (see Table 1). This comparison could point to a mushroom structure with partially overlapped polymer chains or to the formation of a weak brush structure of thickness larger than the Flory radius.

For the first two regimes discussed in Fig. 5, it is hard to extract some information from the ellipsometric investigation because of the few number of experimental points available. The results, however, confirm that at low surface pressures the polymer conformation is two dimensional with $0.1<\Gamma<0.2 \mathrm{mg} \mathrm{m}^{-2}$ significantly higher that the $\Gamma_{2 \mathrm{D}}$ (see Table 1) pointing to the formation of a two dimensional elastic polymer network formed by partially overlapped polymer chains.

\section{Conclusions}

The limiting elasticity of the adsorbed layer of three different Pluronics at the hexane-water interface has been determined by means of dilatational rheology as a function of time and surface pressure.

For the lowest bulk concentrations the limiting elasticity is directly extracted from the real part of the complex dilatational modulus. However, for bulk concentrations above $2 \mathrm{ppm}$ the viscous part of the complex dilatational modulus has a significant influence. Therefore, the limiting elasticity was determined by using a simple diffusional model that fits fairly well with the experimental data.

When the limiting elasticity is plotted against the surface pressure a unique master curve is obtained not depending in the Pluronic bulk concentration. From the location of the maximum and minimum in this plot a picture of the conformational changes of the adsorbed film can be drawn. 
Nulling ellipsometric measurements were carried out to extract more information about the interfacial structure of the adsorbed polymers. It is shown that ellipsometric phase shift values at high surface pressure values can be explained in the basis of a "mushroom" conformation with PPO blocks being partially in both phases.

According to the data obtained from both techniques the following evolution of the adsorbed Pluronic film is proposed (Figure 7). There are four different regions and the surface pressure values where the conformational changes take place are the same for the three Pluronics studied: (I) $\Pi=0-14 \mathrm{mNm}^{-1}$. In this region the scaling law can be applied to determine the parameter $y$. Both, PPO and PEO remain in water. Its value of 2 indicates a two dimendional "stretched chain" conformation. The surface concentration evaluated by ellipsometry seems already above the first overlap surface concentration indicating a partial overlap of polymer chains in 2D.

(II) $\Pi=14-23 \mathrm{mNm}^{-1}$. The PEO blocks start to stretch into the water phase which is the origin of the fall in the elasticity of the adsorbed film due to a mixture of polymers adsorbed in different conformations. The adsorbed layers of Pluronics with higher number of PEO units are more perturbed which lead to smaller elasticity values.

(III) $\Pi=23-31 \mathrm{mNm}^{-1}$. Most of the adsorbed Pluronics are in a three dimensional structure ("mushroom" conformation) compacting the interfacial layer and increasing its elasticity.

(IV) $\Pi>31 \mathrm{mNm}^{-1}$. This region is only observed for the more hydrophobic Pluronics L64 and P9400. The elasticity decreases again which indicate a more disorder structure due to a mixture of polymers conformations. At the water-hexane, this decrease can be related to the protruding of PPO into the hexane phase or to a mixture of polymer mushrooms and brushes. 

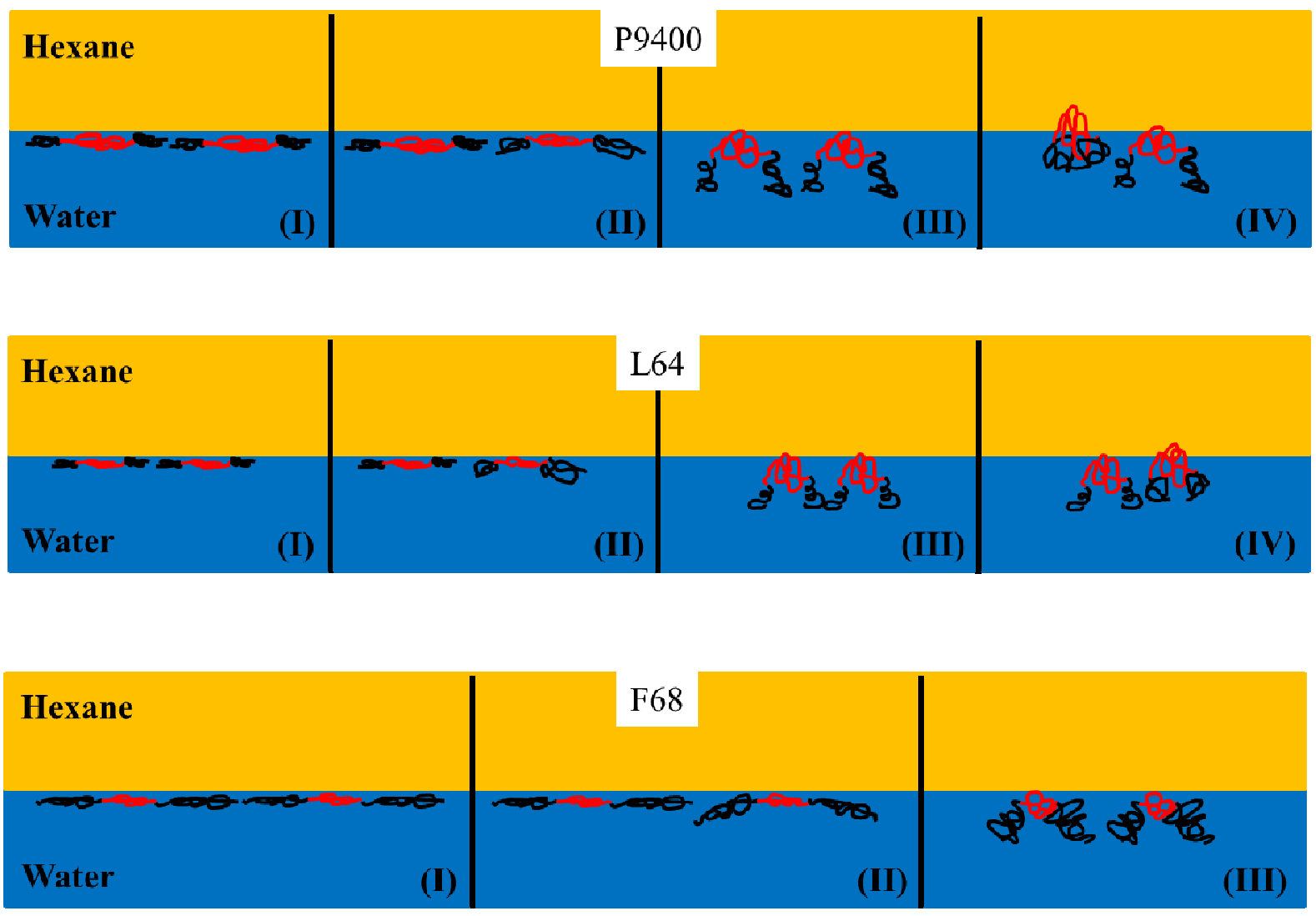

Figure 7. Sketch of the different conformational changes occurring as long as the polymer adsorb at the interface. The red segment stands for PPO units, whereas the black one represents the PEO units. The oil phase is orange and the aqueous phase is blue.

(I) From $\Pi=0-14 \mathrm{mNm}^{-1}$ flat conformation; (II) From $\Pi=14-23 \mathrm{mNm}^{-1}$ mixture of flat and three dimension conformations (PEO protruding into the water); (III) From $\Pi=23-31 \mathrm{mNm}^{-1}$ three dimension "mushroom" conformation; (IV) $\Pi>31 \mathrm{mNm}^{-1}$ disordered structure caused by $P P O$ and PEO interaction.

\section{Acknowledgements}

This study reports part of the results obtained in project CTQ2007-66157PPQ sponsored by the Spanish Ministerio de Ciencia e Innovación and the European Commission (Feder Programme). The authors kindly acknowledge the financial support received. P. R. also acknowledges the financial support of Deutscher Akademischer Austauschdienst (DAAD) and Universidad de Sevilla (IV Plan Propio de Investigación). 
Ramírez Page 29

\section{References}

[1] Alexandridis, P.; Lindman, B. Amphiphilic Block Copolymers: Self-Assembly and Applications; Elsevier, 2000

[2] Berthier, D. L.; Schmidt, I.; Fieber, W.; Schatz, C.; Furrer, A.; Wong, K.; Lecommandoux, S. Langmuir 2010, 26, 7953-7961

[3] Leal-Calderon, F.; Schmitt, V.; Bibette, J. Emulsion Science, Basic Principles; Springer, 2007

[4] Li, X. R.; Li, P. Z.; Zhang, Y. H.; Zhou, Y. X.; Chen, X. W.; Huang, Y. Q.; Liu, Y. Pharm. Res. 2010, 27, 1498-1511

[5] Schramm, L. L. Emulsions, foams and suspensions: fundamentals and applications; 2005

[6] Tadros, T., Polymeric Surfactants in: Novel Surfactants. Holmberg, K. (Eds.), Marcel Dekker, New York, 2003

[7] Tadros, T. Applied Surfactants, Principles and Applications; Wiley-VCH, Weinheim, 2005

[8] Tavano, L.; Muzzalupo, R.; Trombino, S.; Cassano, R.; Pingitore, A.; Picci, N. Colloids Surf., $B$ 2010, 79, 227-234

[9] Zoller, U. Handbook of Detergents. Part E: Applications; CRC Press, 2009

[10] Aguié-Béghin, V.; Leclerc, E.; Daoud, M.; Douillard, R. J. Colloid Interface Sci. 1999, 214, $143-155$

[11] Roger, D. Colloids Surf., B 1993, 1, 333-340

[12] Bai, Z.; Lodge, T. P. Langmuir 2010, 26, 8887-8892

[13] Morales, M. A.; Jain, T. K.; Labhasetwar, V.; Leslie-Pelecky, D. L. J. Appl. Phys. 2005, 97, 10Q905 
[14] Blomqvist, B. R.; Warnheim, T.; Claesson, P. M. Langmuir 2005, 21, 6373-6384

[15] Hambardzumyan, A.; Aguie-Beghin, V.; Daoud, M.; Douillard, R. Langmuir 2004, 20, 756763

[16] Kim, C.; Yu, H. Langmuir 2003, 19, 4460-4464

[17] Monroy, F.; Ortega, F.; Rubio, R. G.; Noskov, B. A., Surface Rheology Studies of Spread and Adsorbed Polymer Layers in: Interfacial Rheology. Miller, R.;Liggieri, L. (Eds.), Brill, Leiden, 2009

[18] Munoz, M. G.; Monroy, F.; Ortega, F.; Rubio, R. G.; Langevin, D. Langmuir 2000, 16, 1083-1093

[19] Munoz, M. G.; Monroy, F.; Ortega, F.; Rubio, R. G.; Langevin, D. Langmuir 2000, 16, 1094-1101

[20] Noskov, B. A. Curr. Opin. Colloid Interface Sci. 2010, 15, 229-236

[21] Noskov, B. A.; Akentiev, A. V.; Loglio, G.; Miller, R. J. Phys. Chem. B 2000, 104, 79237931

[22] Noskov, B. A.; Lin, S. Y.; Loglio, G.; Rubio, R. G.; Miller, R. Langmuir 2006, 22, 26472652

[23] Sedev, R.; Steitz, R.; Findenegg, G. H. Physica B 2002, 315, 267-272

[24] Vieira, J. B.; Li, Z. X.; Thomas, R. K. J. Phys. Chem. B 2002, 106, 5400-5407

[25] Vieira, J. B.; Li, Z. X.; Thomas, R. K.; Penfold, J. J. Phys. Chem. B 2002, 106, 1064110648

[26] Leclerc, E.; Daoud, M. Macromolecules 1997, 30, 293-300

[27] Munoz, M. G.; Monroy, F.; Hernandez, P.; Ortega, F.; Rubio, R. G.; Langevin, D. Langmuir 2003, 19, 2147-2154 
[28] Ramírez, P.; Muñoz, J.; Fainerman, V. B.; Aksenenko, E. V.; Mucic, N.; Miller, R. Colloids Surf., A 2011, 391, 119-124

[29] Roger, D. Colloids Surf., A 1994, 91, 113-119

[30] Exerowa, D.; Gotchev, G.; Kolarov, T.; Kristov, K.; Levecke, B.; Tadros, T. Colloids Surf., A 2009, 335, 50-54

[31] Gotchev, G.; Kolarov, T.; Khristov, K.; Exerowa, D. Adv. Colloid Interface Sci. In Press, Corrected Proof,

[32] Gotchev, G.; Kolarov, T.; Khristov, K.; Exerowa, D. Colloids Surf., A 2010, 354, 56-60

[33] Hansen, F. K. Langmuir 2008, 24, 189-197

[34] Alexandridis, P.; Holzwarth, J.F.; Hatton, T.A. Macromolecules 1994, 27, 2414-2425.

[35] Loglio, G.; Pandolfini, P.; Miller, R.; Makievski, A. V.; Ravera, F.; Ferrari, M.; Liggieri, L., Drop and Bubble Shape Analysis as Tool for Dilatational Rheology of Interfacial Layers in: Novel Method to Study Interfacial Layers. Möbius, D.;Miller, R. (Eds.), Elsevier, 2001

[36] Zholob, S. A.; Kovalchuk, V. I.; Makievski, A. V.; Krägel, J.; Fainerman, V. B.; Miller, R., Determination of the Dilatational Elasticity and Viscosity from the Surface Tension Response to Harmonic Area Perturbations in: Interfacial Rheology. Miller, R.;Liggieri, L. (Eds.), Brill, Leiden, 2009

[37] Lucassen, J.; Van den Tempel, M. Chem. Eng. Sci. 1972, 27, 1283-1291

[38] Azzam, R.M.A.; Bashara, N.M. Ellipsometry and Polarised Light, North-Holland Publishing Co., 1977

[39] Stocco, A.; Tauer, K.; Pispas, S.; Sigel, R. J. Colloids Int. Sci. 2011, 355, 1, 172-178

[40] Lekner, J. Theory of Reflection, Martinus Nijhoff, Dordrecht, The Netherlands, 1987

[41] Russev, S.C.; Arguirov, T.V.; Gurkov, T.D. Colloids Surf., B: Biointerfaces 2000, 19, 89 
[42] Stocco, A.; Tauer, K. Eur. Phys. J. E 2009, 30 4, 431-438

[43] de Gennes, P. G. Macromolecules 1980,13,1069-1075

[44] Georgieva, D.; Schmitt, V.; Leal-Calderon, F.; Langevin, D. Langmuir 2009, 25, $5565-5573$

[45] Wulff-Pérez, M.; Torcello-Gómez, A.; Martín-Rodríguez, A.; Gálvez-Ruiz, M.J.; de Vicente, J. Food Hydrocolloids 2011, 25, 677-686

[46] Day, S.P.R.; Bain, C.D. Phys. Rev. E, 2007, 76, 041601.

\section{Legends}

Figure 1. Lateral and top view of the model proposed to calculate the overlap surface concentration $\left(\Gamma_{2 \mathrm{D}}\right)$, for the triblock copolymers in a two dimensional conformation.

Figure 2. Storage (E', solid symbols) and loss (E', open symbols) modulus as a function of frequency. a) Pluronic L64, c = 0.1 ppm. b) Pluronic P9400, c = 0.1 ppm. c) Pluronic LF68, c = $0.1 \mathrm{ppm}$

Figure 3. Storage (E', solid symbols) and loss (E', open symbols) modulus as a function of frequency. a) Pluronic L64, c = 10 ppm (triangles) and 20 ppm (squares); b) Pluronic P9400, c = $10 \mathrm{ppm}$ (triangles) and $20 \mathrm{ppm}$ (squares); c) Pluronic F68, c = $10 \mathrm{ppm}$ (triangles) and $20 \mathrm{ppm}$ (squares). The lines are the best fit of the experimental data to the diffusional model with the values of $\mathrm{E}_{0}$ and $\omega_{0}$ given in Table 1.The standard deviation of the experimental $E^{\prime}$ and $E^{\prime}$ values was about $\pm 1 \mathrm{mNm}^{-1}$. (see Supplementary Information). 
Figure 4. Limiting elasticity as a function of time. a) Pluronic L64, c $=0.01 \mathrm{ppm}$ (triangles), 0.1 ppm (squares) and $2 \mathrm{ppm}$ (circles); b) Pluronic P9400, c $=0.01 \mathrm{ppm}$ (triangles) $0.1 \mathrm{ppm}$ (squares) and $2 \mathrm{ppm}$ (circles); c) Pluronic F68, c = $0.01 \mathrm{ppm}$ (triangles), $0.1 \mathrm{ppm}$ (squares) and 2 ppm (circles).

Figure 5. Limiting elasticity as a function of surface pressure. a) Pluronic L64, b) Pluronic P9400 and c) Pluronic F68. The open symbols are the data for concentrations below c $=2 \mathrm{ppm}$ where the limiting elasticity was directly obtained from the storage modulus, whereas the filled symbols account for the highest concentrations where the diffusional model has been used to determine $\mathrm{E}_{0}$. The solid lines show a linear dependence of $\mathrm{E}_{0}$ on $\Pi$ at low surface pressures, $\mathrm{E}_{0}=2 \Pi$. The dotted lines separate the four different conformational regions.

Figure 6. Experimental ellipsometric phase shift values $\left(\Delta-\Delta_{0}\right)$ as a function of surface pressure $\Pi$ (bottom x-axis) for L64, P9400 and F68 Pluronics. Dashed, dotted and solid (red) lines are the simulated $\Delta-\Delta_{0}$ as a function of surface concentration $\Gamma$ (top x-axis) for three polymer conformations: "flat", "coil" and "mushroom" accounting for a fraction f of PPO in hexane respectively. The dash-dotted (black) lines are the simulated $\Delta-\Delta_{0}$ as a function of surface pressure (bottom x-axis) accounting for the capillary wave profile. The solid thin lines help the reader to find the surface concentration of a given model starting from the experimental data and accounting for the capillary wave contribution. The standard deviation of the experimental data was about $\pm 0.1^{\circ}$. 
Figure 7. Sketch of the different conformational changes occurring as long as the polymer adsorb at the interface. The red segment stands for PPO units, whereas the black one represents the PEO units. The oil phase is orange and the aqueous phase is blue.

(I) From $\Pi=0-14 \mathrm{mNm}^{-1}$ flat conformation; (II) From $\Pi=14-23 \mathrm{mNm}^{-1}$ mixture of flat and three dimension conformations (PEO protruding into the water); (III) From $\Pi=23-31 \mathrm{mNm}^{-1}$ three dimension "mushroom" conformation; (IV) $\Pi>31 \mathrm{mNm}^{-1}$ disordered structure caused by PPO and PEO interaction. 


\section{Tables}

Table 1. Molecular weight of the three Pluronics and overlap polymer surface concentrations.

\begin{tabular}{|c|c|c|c|c|}
\hline & $\mathrm{M}_{\mathrm{w}} / \mathrm{g} \cdot \mathrm{mol}^{-1}$ & $\Gamma_{2 \mathrm{D}}\left[\mathrm{mg} / \mathrm{m}^{2}\right]$ & $\Gamma_{\mathrm{PPO}}\left[\mathrm{mg} / \mathrm{m}^{2}\right]$ & $\Gamma_{\mathrm{PEO}}\left[\mathrm{mg} / \mathrm{m}^{2}\right]$ \\
\hline L64 & 2900 & 0.032 & 0.1 & 0.31 \\
\hline P9400 & 4600 & 0.023 & 0.09 & 0.28 \\
\hline F68 & 8400 & 0.037 & 0.29 & 0.11 \\
\hline
\end{tabular}


Table 2. Limiting elasticity, $E_{0}$ and characteristic frequency, $\omega_{0}$, used to fit the experimental data to the diffusional model for the highest Pluronic bulk concentrations (10 and $20 \mathrm{ppm}$ ).

\begin{tabular}{|c|c|c|c|c|}
\cline { 2 - 5 } \multicolumn{1}{c|}{} & \multicolumn{4}{c|}{ Concentration [ppm] } \\
\cline { 2 - 5 } & \multicolumn{3}{c|}{10} & \multicolumn{2}{c|}{20} \\
\cline { 2 - 5 } & $E_{0}\left[\mathrm{mNm}^{-1}\right]$ & $\omega_{0}\left[\mathrm{~s}^{-1}\right]$ & $E_{0}\left[\mathrm{mNm}^{-1}\right]$ & $\omega_{0}\left[\mathrm{~s}^{-1}\right]$ \\
\hline L64 & 14.5 & 0.03 & 14 & 0.08 \\
\hline P9400 & 16.5 & 0.015 & 14.5 & 0.03 \\
\hline F68 & 19.5 & 0.002 & 20 & 0.013 \\
\hline
\end{tabular}

